\title{
Harmonic Diffeomorphisms and Conformal Distortion of Riemann Surfaces
}

\begin{abstract}
VLADIMIR MARKOVIC
In this paper we study the change of conformal structure induced by harmonic diffeomorphisms between Riemann surfaces. The main result of this paper is to answer the following question raised by R. Schoen (see [20]): Is it true that Riemann surfaces which are related by a surjective harmonic diffeomorphism are necessarily quasiconformally related? We show that there exists a pair of Riemann surfaces of infinite topological type, which are related by a surjective harmonic diffeomorphism but which are not quasiconformally related. Also we characterize when the above question has a positive answer in the case of Riemann surfaces of finite topological type.
\end{abstract}

\section{Introduction.}

Suppose that $R$ and $S$ are two Riemann surfaces. In addition, assume that surface $S$ is of hyperbolic type. That is the universal covering surface of $S$ is the unit disc. Denote by $\rho^{2}|d w|^{2}$ the Poincaré (or the hyperbolic) metric on $S$. Let $f: R \rightarrow S$, be a map of the class $C^{2}$. We say that $f$ is a harmonic map of the surface $R$ into the surface $S$ with respect to the hyperbolic metric (in the rest of the paper just a harmonic map) if the following equation is satisfied

$$
f_{z \bar{z}}+2(\log \rho)_{w}(f(z)) f_{z} f_{\bar{z}}=0,
$$

where $\mathrm{z}$ and w are local parameters on $R$ and $S$ respectively and $\rho^{2}$ is the function of $w$. If map $f$ is univalent we say that $f$ is a harmonic diffeomorphism. If $f$ is also surjective we say that $f$ is a surjective harmonic diffeomorphism. All maps considered in this paper are of positive orientation.

The following question was raised in [20].

Question 1 (Schoen). Is it true that Riemann surfaces which are related by a surjective harmonic diffeomorphism are necessarily quasiconformally related? 
In other words this means that if $f: R \rightarrow S$ is a surjective harmonic diffeomorphism of $R$ onto $S$ the question is whether we can find a quasiconformal map $g: R \rightarrow S$. Quasiconformal maps are often used to measure the conformal distortion between Riemann surfaces. A Harmonic diffeomorphism $f$ does not have to be a quasiconformal map. Still, we want to know whether the change of conformal structure obtained by $f$ is bounded in the sense of the Teichmüller distance. The Teichmüller distance in a natural way measures the distance between conformal structures.

The test case proposed by Schoen is as follows. Take $R=\mathbf{C}$ and $S=\mathbf{D}$ where $\mathbf{C}$ is the complex plane and $\mathbf{D}$ is the unit disc. It is well known that there is no quasiconformal map from $\mathbf{C}$ onto $\mathbf{D}$ or vice versa. So in order to study the above question, in this case we need to investigate whether there is a harmonic diffeomorphism from $\mathbf{C}$ onto $\mathbf{D}$. This special case is a hard open problem and it has not been solved yet. But in the past 10 years several papers have been published in connection with this case. At present, the methods developed to study this case require certain assumptions on the growth of the map $f$ near infinity. In particular it was proved in [10] and [11] that if the Hopf differential of the diffeomorphism $f$ is a polynomial then the map $f$ is not surjective. In the recent paper [6] the authors considered the case that the Hopf differential of the diffeomorphism $f$ does not have to be a polynomial but rather to belong to some more general class of the entire functions. They showed that for a large class of entire functions the corresponding diffeomorphism $f$ is not surjective. Also in [3] it was proved that under certain assumptions on the growth of the energy of the harmonic diffeomorphism $f: \mathbf{C} \rightarrow \mathbf{D}$ the map $f$ is not surjective.

The main result of this paper is to give the answer to Question 1.

Theorem 1.1. There exist two Riemann surfaces $R$ and $S$ which are related by a surjective harmonic diffeomorphism and which are not quasiconformally related.

Remark 1.1. One advantage of our example is that the diffeomorphism $f$ meets the requirement that $\left(R,|\partial f|^{2}|d z|^{2}\right)$ is a complete Riemannian surface. Therefore $|\partial f|^{2}$ is the canonical solution of the equation (2.3) (see Section 2).

We prove this theorem by constructing an explicit example of surfaces $R, S$ and a surjective harmonic diffeomorphism $f: R \rightarrow S$ such that $R$ and $S$ are not quasiconformally related. The surfaces in our example are of 
infinite topological type(infinitely generated fundamental group). It is not hard to imagine that besides the example we offer here there are many other examples of similar nature, once we restrict to Riemann surfaces of infinite topological type.

Let us roughly illustrate the idea for the construction of this example. Set $S=\mathbf{C} \backslash \mathbf{Z}$, where $\mathbf{Z}$ stands for all integer points on the real line in the complex plane $\mathbf{C}$. The planar domain $S$ is a hyperbolic Riemann surface. There are many harmonic diffeomorphisms defined on $S$. In particular there are many harmonic diffeomorphisms $f: S \rightarrow R$ such that $R$ is a punctured plane domain homeomorphic to $S$ and such that $f$ is symmetric with respect to the real axis. It is clear that every such diffeomorphism $f$ extends over the punctures to the map defined on the whole complex plane(we call this extension $f$ again and it is not necessarily surjective). In this paper we will show that the map $f$ (which is harmonic on $S$ ) can be chosen to be a surjective diffeomorphism of the whole complex plane and still that $R$ and $S$ are not quasiconformally related. One of the issues is to find a criteria under which the diffeomorphism $f$ is a surjective map. We resolve this by applying the recent results of Brakalova and Jenkins about the extended version of the solution to the Beltrami equation (see [7], and Section 2. of the present paper). In particular we can construct the map $f$ with the above properties and such that

$$
\limsup _{n \rightarrow \infty} \frac{f(n+1)-f(n)}{f(n)-f(n+1)}=\infty .
$$

Also we can construct the map $f$ such that the expression in (1.1) approaches $\infty$ very slowly, and in particular slow enough such that $f$ remains a surjective map. On the other hand, we will prove that the condition (1.1) implies that the domains $S$ and $R$ are not quasiconformally related.

Still we hope that we can find an appropriate class of surfaces such that the Question 1. has the positive answer. We believe that the class of Riemann surfaces of finite topological type is a proper choice. It is well known that every Riemann surface of finite topological type is conformally equivalent to a closed surface (of finite genus) with a finite number of discs and punctures removed. If we consider only closed surfaces, M.Wolf proved (see [25]) that in the homotopy class of an arbitrary homeomorphism between closed Riemann surfaces there exists a harmonic diffeomorphism which is necessarily a quasiconformal map. Furthermore there is a natural parameterization of Teichmüller space by harmonic diffeomorphisms. From the point of view of Question 1. Wolf's theorems (see also [25], [26] for further 
results) show that the amount of distortion which can be obtained by a harmonic diffeomorphism is bounded in the sense of the Teichmüller metric. This provides the positive answer to Question 1. If in addition we remove certain number of discs and punctures from a closed surfaces we have a new type of situation. One such case is the above special case proposed by Schoen, where the considered Riemann surfaces are $\mathbf{C}$ and $\mathbf{D}$. Another test case similar to the one proposed by Schoen is as follows. For $0<r<1$ we set $\mathbf{D}^{*}=\mathbf{D} \backslash\{0\}$ and $P(r)=\{r<|z|<1\}$. We know that there is no quasiconformal map of $\mathbf{D}^{*}$ onto $P(r)$. The question is whether there is a surjective harmonic diffeomorphism of $\mathrm{D}^{*}$ onto $P(r)$. As it turns out there is no essential difference in considering this special case and any other case where surfaces of finite topological type are involved. This conclusion is summarized in the following theorem.

Theorem 1.2. Suppose that for every $0<r<1$ there is no surjective harmonic diffeomorphism of the domain $D^{*}$ onto the domain $P(r)$. Let $f: R \rightarrow S$ be a surjective harmonic diffeomorphism between any two Riemann surfaces $R$ and $S$ of finite topological type, which satisfies the condition that $\left(R,|\partial f|^{2}|d z|^{2}\right)$ is a complete metric space Then $R$ and $S$ are quasiconformally related.

Query. Is it true that if there is no surjective harmonic diffeomorphism from the complex plane onto the unit disc then every two Riemann surfaces of finite topological type which are related by a surjective harmonic diffeomorphism are necessarily quasiconformally related.

The rest of the paper is organized as follows. In Section 2. we recall and prove some facts about harmonic and quasiconformal maps. In Section 3. we construct a pair of planar domains of infinite connectivity to prove Theorem 1.1. In Section 4. we bring into the discussion surfaces of finite topological type and prove Theorem 1.2

\section{Preliminary Results.}

In this section we recall or prove certain facts about harmonic diffeomorphisms and quasiconformal maps which are needed for the proof of Theorem 1.1 and Theorem 1.2.

Suppose that $R$ is a hyperbolic Riemann surface and $f: R \rightarrow S$ a 
harmonic diffeomorphism(not necessarily surjective). Set

$$
H o p f(f)=\left(\rho^{2} \circ f\right) f_{z} \overline{\left(f_{\bar{z}}\right)} d z^{2} .
$$

Here $\rho^{2}$ denotes the density of the hyperbolic metric on $S$. The expression $H o p f(f)$ is called the Hopf differential associated to the map $f$. It is well known that since $f$ is a harmonic map $\operatorname{Hop} f(f)$ is a holomorphic quadratic differential on the surface $R$. By $Q D(R)$ we denote the space of all holomorphic quadratic differentials on $R$. We also define

$$
\partial f=(\rho \circ f) f_{z}, \bar{\partial} f=(\rho \circ f) f_{\bar{z}}
$$

and

$$
\operatorname{Belt}(f)=\frac{f_{\bar{z}}}{f_{z}} \frac{d \bar{z}}{d z}
$$

The expressions $\partial f$ and $\bar{\partial} f$ are partial derivatives taken with respect to the hyperbolic metric on the image surface $S$ and $B e l t(f)$ is the Beltrami dilatation of the map $f$. We set $\operatorname{Hop} f(f)=\psi d z^{2}$ and Belt $(f)=\mu \frac{d \bar{z}}{d z}$. The following relation is the Bochner identity for harmonic maps

$$
\Delta \log |\partial f|=|\partial f|^{2}-\left|\frac{\psi^{2}}{\left|\partial f^{2}\right|}\right|
$$

where $\Delta$ denotes the ordinary Laplacian $\partial \bar{\partial}$ operator on Riemann surface. Set

$$
\tau=\log \frac{1}{|\mu|} .
$$

We have an equivalent version of identity (2.1)

$$
\Delta \tau=|\psi| \sinh (\tau)
$$

We refer to [21] for the background on harmonic maps.

In order to prove Theorem 1.1 we need to directly construct harmonic diffeomorphisms. Unlike the functions which are harmonic with respect to the Euclidean metric, in this hyperbolic setting there are no known representation formulas for harmonic maps similar to those which apply to ordinary harmonic functions. This makes the construction more difficult. But it turned out that we are still able to produce harmonic diffeomorphisms. In [24] T. Wan proved (see also [5], [22]) the following result 
Proposition 2.1 (Wan). Suppose that $R$ is a hyperbolic Riemann surface with the hyperbolic metric $\sigma^{2}\left|d z^{2}\right|$. Let $\psi d z^{2} \in Q D(R)$. Then there is a unique positive solution $\omega$ of the equation

$$
\Delta \log \omega=|\omega|^{2}-\left|\frac{\psi^{2}}{\omega^{2}}\right|,
$$

such that the metric space $\left(R,|\omega|^{2}\left|d z^{2}\right|\right)$ is a complete metric space and such that $\sigma^{-2} \omega \geq 1$ holds on $R$. In addition if $\gamma$ is any other solution of the equation (2.3) then the relation $\gamma \leq \omega$ holds on $R$.

Moreover if the Riemann surface $R$ is the unit disc $\mathbf{D}$ then there is a unique harmonic diffeomorphism (not necessarily surjective) $f: \mathbf{D} \rightarrow \mathbf{D}$ such that $\operatorname{Hop} f(f)=\psi d z^{2}$ and $|\partial f|=\omega$.

Remark 2.1. In the rest of the paper we will often use Proposition 2.1, and in particular the existence of the solution of the equation (2.3) for a given differential $\psi d z^{2}$. When we refer to the solution $\omega$ of the equation (2.3) we will always mean the unique solution which satisfies the condition that $\left(R,|\omega|^{2}|d z|^{2}\right)$ is a complete metric space.

The previous proposition guarantees the existence of a harmonic diffeomorphism for a given Hopf differential in the case of the unit disc. The map $f$ need not to be surjective. It is likely that one can extend this last part of the Proposition 2.1 to the case of arbitrary hyperbolic Riemann surfaces. This means that if $R$ is an arbitrary hyperbolic Riemann surface and $\psi d z^{2} \in Q D(R)$ then there exists a Riemann surface $S$ which is homeomorphic to $R$ and a harmonic diffeomorphism $f: R \rightarrow S$ whose Hopf differential is $\psi d z^{2}$. Also $f(R)$ is a subsurface of $S$ with the property that the the natural inclusion map of the fundamental group of $f(R)$ into the fundamental group of $S$ is isomorphism between these groups. For the existence of a harmonic diffeomorphism for a given Hopf differential in the case when the Riemann surface $R$ is the complex plane we refer to [8], [2], [5]. In this paper we only need the following corollary of Proposition 2.1 and the Uniformization theorem.

Corollary 2.1. Let $\mathbf{D}^{*}$ be the punctured unit disc and let $\psi d z^{2} \in Q D\left(\mathbf{D}^{*}\right)$. Then there is a hyperbolic Riemann surface $S$ which is homeomorphic to bf $D^{*}$ and there is a harmonic diffeomorphism (not necessarily surjective) $f: \mathbf{D}^{*} \rightarrow S$ with $\operatorname{Hop} f(f)=\psi d z^{2}$ and which satisfies that $|\partial f|=\omega$ is the solution to the equation (2.3). Also the natural inclusion map of the fundamental group of the subsurface $f\left(\mathbf{D}^{*}\right)$ into the fundamental group of $S$ is isomorphism between these groups. 
Proof. Denote by $\varphi d w^{2}$ the lift the differential $\psi d z^{2}$ onto the upper half plane $\mathbf{H}^{+}$, obtained by the universal covering map $\pi: \mathbf{H}^{+} \rightarrow \mathbf{D}^{*}$. Here by $w$ we denote the complex parameter on $\mathbf{H}^{+}$. We may assume that the group $G$ of the covering transformation acting on the upper half plane is the cyclic group generated by the translation $T(w)=w+1$. We apply the Proposition 2.1 onto the lifted differential and let $g$ be the resulting harmonic diffeomorphism of the upper half plane onto itself. The map $g$ does not have to be surjective. As we said before, since $|\partial g|$ is the solution of the equation (2.3), the uniqueness implies that the Beltrami dilatation $\mu=\operatorname{Belt}(g)$ satisfies the condition $\mu(T(w))=\mu(w)$. So if $B$ is defined by

$$
B=f \circ T \circ f^{-1}
$$

we have that $B$ is a conformal map $B: f\left(\mathbf{H}^{+}\right) \rightarrow \mathbf{H}^{+}$. But we also know that $B \circ f$ is a harmonic map with respect to the hyperbolic metric on the upper half plane. Therefore (see also [25], [23]) $B$ has to be an isometry with respect to the hyperbolic metric and consequently a Moebius transformation. Also it is clear that $B$ can not fix any point inside the upper half plane. We conclude that the cyclic group $G_{1}$ generated by $B$ is a covering group of the hyperbolic Riemann surface $S$. The corollary now follows from the fact that $B=f \circ T \circ f^{-1}$.

Recall that in this section we are assuming that $R$ is a hyperbolic Riemann surface with the hyperbolic density $\sigma^{2}|d z|^{2}$. The Bers space $B Q D(R)$ is a subspace of $Q D(R)$. The differential $\psi d z^{2}$ is said to belong to $B Q D(R)$ if

$$
\|\psi\|_{B}=\sup _{p \in R}\left(\sigma^{-2}|\psi|\right)(p) \leq \infty .
$$

The Bers space is a Banach space with the Bers norm $\|\psi\|_{B}$. Note that $\left(\sigma^{-2}|\psi|\right)(p)$ is a well defined function on $R$. The following proposition is proved in [24].

Proposition 2.2. If $R$ is a hyperbolic Riemann surface and $\psi d z^{2} \in$ $B Q D(R)$ then there is a Riemann surface $S$ and a surjective harmonic diffeomorphism $f: R \rightarrow S$ such that $H o p f(f)=\psi d z^{2}$ and $|\partial f|=\omega$, where $\omega$ is a solution to the equation (2.3) from Proposition 2.1. Moreover the map $f$ is a quasiconformal diffeomorphism, that is we have

$$
\|\operatorname{Belt}(f)\|_{\infty}<1 \text {. }
$$

Under the above assumptions the map $f$ is uniquely determined. 
The construction of a harmonic diffeomorphism for a given Hopf differential, it based on a method which establishes the connection between quadratic differentials on $R$ and the Gauss maps of cross cuts in the Minkowski space. But in order to study Question 1. we need a criteria when the constructed harmonic map is actually surjective. In search for a proper criteria we analyze the well known Beltrami equation

$$
f_{\bar{z}}=\mu f_{z} .
$$

The idea for the proof of Theorem 1.1 is to construct a harmonic diffeomorphism for the given Hopf differential $\psi d z^{2}$ from $Q D(R)$ by properly solving the corresponding Beltrami equation. Since in this case we loose the condition that $\|\mu\|_{\infty}<1$, we can not apply the classical solution of the Beltrami equation. Instead, as the sufficient conditions for surjectivity we use the results of Brakalova and Jenkins [7]. Under the appropriate assumptions these show that there is a proper solution to the Beltrami equation even when we have that $\|\mu\|_{\infty}=1$ (see Theorem 1, Proposition 3, and Proposition 5 in $[7])$.

Proposition 2.3 (Brakalova-Jenkins). Suppose that $\mu \in L^{\infty}(\mathbf{C})$, $\|\mu\|_{\infty} \leq 1$, satisfies the following two conditions:

(1) For $r \rightarrow \infty$

$$
\int_{\{|z|<r\}} \frac{1}{1-|\mu|}=O\left(r^{2}\right) .
$$

(2) For each bounded measurable set $B$ in $\mathbf{C}$

$$
\int_{B} \exp \frac{\frac{1}{1-|\mu|}}{1+\log \frac{1}{1-|\mu|}}<\infty
$$

Then there exists a homeomorphism $f: \mathbf{C} \rightarrow \mathbf{C}$ which is $A C L$ and whose partial derivatives are in $L^{q}$ on every compact subset in plane for each $q<2$. The map $f$ satisfies the Beltrami equation

$$
f_{\bar{z}}=\mu f_{z} .
$$

Under the above assumptions the map $f$ is uniquely determined up to the postcomposition with a Moebius transformation of the complex plane onto itself. 
In the above proposition the conclusion that the map $f$ is ACL means that $f$ is absolutely continuous on lines and therefore it is differentiable almost everywhere in the plane. As it is pointed out in [7] the condition (2.5) is essential for the map $f$ to be surjective. In addition to the uniqueness part of the above proposition the fact that the map $f$ is surjective is essential for our application. A suitable corollary of Proposition 2.3 from the point of view of our paper is the following.

Corollary 2.2. Suppose that $\mu$ is a continuous function in $\mathbf{C}$ which satisfies $\|\mu\|_{\infty} \leq 1$ and $|\mu|<1$ on every compact set in the complex plane. If $\mu$ satisfies the growth condition

$$
\int_{\{|z|<r\}} \frac{1}{1-|\mu|}=O\left(r^{2}\right),
$$

when $r \rightarrow \infty$ then there exists a homeomorphism $f: \mathbf{C} \rightarrow \mathbf{C}$ which is $A C L$ and whose partial derivatives are in $L^{q}$ on every compact subset in plane for each $q<2$. The map $f$ satisfies the Beltrami equation

$$
f_{\bar{z}}=\mu f_{z}
$$

Under the above assumptions the map $f$ is uniquely determined up to the postcomposition with a Moebius transformation of the complex plane onto itself.

Proof. Since $\mu$ is a continuous function and $|\mu|<1$ on every compact set in $\mathbf{C}$ we see that the condition (2.6) is automatically satisfied. The rest of proof follows from Proposition 2.3.

We will also need a result about the continuity of the solutions of the equation (2.3). The following lemma is essentially proved in [24] (see also [22]) but for the readers convenience we give the short proof.

Lemma 2.1. With the notation stated in Proposition 2.1 we have the following. Let $R$ be a hyperbolic Riemann surface and suppose that $\psi_{n} d z^{2} \in$ $Q D(R)$ is a sequence of quadratic differentials on $R$ which converges to $\psi d z^{2}$ locally uniformly on compact sets in $R$. If $\omega_{n}$ and $\omega$ are the solutions of the equation (2.3) for $\psi_{n} d z^{2}$ and $\psi d z^{2}$ respectively, then the sequence of functions $\omega_{n}$ converges to the function $\omega$ uniformly on compact sets in $R$. 
Proof. We prove this lemma in the case when the Riemann surface $R$ is the unit disc. The general case then follows at once from the Uniformization theorem and the uniqueness part of Proposition 2.1.

Set

$$
\varphi_{r}(z)=\psi(r z), \varphi_{n, r}(z)=\psi_{n}(r z), z \in \mathbf{D}
$$

for $0<r<1$. Note that both $\varphi_{n, r}$ and $\varphi_{r}$ are in $B Q D(\mathbf{D})$ for every $r$. Now fix $r<1$. Set

$$
\left\|\varphi_{n, r}-\varphi_{r}\right\|_{B}=\epsilon_{n, r} .
$$

We have that $\epsilon_{n, r} \rightarrow 0$ when $n \rightarrow 0$. Let $\omega_{n, r}, \omega_{r}$ stand for the solutions of the equation (2.3) for $\varphi_{n, r}$ and $\varphi_{r}$ respectively. It was proved in [24], [22] that $\omega_{n, r} \rightarrow \omega_{r}$ uniformly on compact sets in $\mathbf{D}$ for fixed $r$ and when $n \rightarrow \infty$.

On the other hand it is known (see Theorem 12 in [24]) that for fixed $n$ and when $r$ approaches 1 we have $\omega_{n, r} \rightarrow \omega_{n}$ and $\omega_{r} \rightarrow \omega$ uniformly on compact sets in $\mathbf{D}$. Now we can choose (by the standard diagonal trick) the sequence $\left\{r_{n}\right\}, r_{n} \rightarrow 1$ such that $\left|\omega_{n, r_{n}}-\omega_{n}\right| \rightarrow 0$ and $\left|\omega_{n, r_{n}}-\omega\right| \rightarrow 0$ on every compact set in $\mathbf{D}$.

So far in this section we have stated results in connection with existence and surjectivity of harmonic and quasiconformal maps. For the purposes of the proof of Theorem 1.1 we also need a criteria which guarantees that the two homeomorphic planar domains (see the definition below) are not quasiconformally related. In the rest of this section we consider the following situation. Let $R=\mathbf{C} \backslash \mathbf{Z}$ where $\mathbf{Z}$ is a set of all integers on the real line R. Let $f: \mathbf{C} \rightarrow \mathbf{C}$ be a diffeomorphism (not necessarily harmonic) of the complex plane onto itself which preserves $\mathbf{R}$. Set $a_{k}=f(k)$ for each $k \in \mathbf{Z}$, $A=f(\mathbf{Z})$ and $S=\mathbf{C} \backslash A$. Then $f$ is a diffemorphism of $R$ onto $S$. Since $f$ preserves the real line we have that $a_{k}<a_{(k+1)}$ for each $k$. Let

$$
\mathbf{s}=\sup _{k \in \mathbf{Z}}\left|\frac{a_{(k+1)}-a_{k}}{a_{k}-a_{(k-1)}}\right|+\left|\frac{a_{(k-1)}-a_{k}}{a_{k}-a_{(k+1)}}\right| .
$$

We prove

Lemma 2.2. With the notation stated above we have that if the supremum $\mathbf{s}$ given by (2.8) is equal $\infty$ then the planar domains $R$ and $S$ are not quasiconformally related. 
Proof. The proof is by a contradiction. Let us assume that $\mathbf{s}=\infty$ and that there is a K-quasiconformal map $g: R \rightarrow S$, for some $K>1$. Of course, the map $g$ does not have to satisfy the same boundary condition as the diffeomorphism $f$. Clearly the map $g$ extends to the quasiconformal map of the complex plane onto itself. From the assumption $\mathbf{s}=\infty$, with no loss of generality, we conclude that there is a sequence of integers $k_{n}, n \in \mathbf{N}$, such that

$$
\left|\frac{a_{\left(k_{n}+1\right)}-a_{k_{n}}}{a_{k_{n}}-a_{\left(k_{n}-1\right)}}\right| \rightarrow 0, n \rightarrow \infty .
$$

When we say that we do not loose on generality that means that since $\mathbf{s}=\infty$ we can find a sequence $a_{k_{n}}$ such that either one of the four similar cases to (2.9) has to occur. All of them are to be treated in the same way as we will do with (2.9).

Denote by $p_{n}$ and $q_{n}$ the integers such that $g\left(p_{n}\right)=a_{k_{n}}$ and $g\left(q_{n}\right)=$ $a_{\left(k_{n}+1\right)}$. Next we define the maps $h_{n}: \mathbf{C} \rightarrow \mathbf{C}, n \in \mathbf{N}$ by

$$
h(z)=\frac{g\left(\left(q_{n}-p_{n}\right) z+p_{n}\right)-a_{k_{n}}}{a_{\left(k_{n}+1\right)}-a_{k_{n}}}, z \in \mathbf{C} .
$$

Each $h_{n}$ is a K-quasiconformal map and $h_{n}(0)=0, h_{n}(1)=1$ for each $n$. Also set

$$
\mathbf{Z}(n)=\left\{\frac{k}{q_{n}-p_{n}}: k \in \mathbf{Z}\right\}
$$

and

$$
A(n)=\left\{\frac{a_{k}-a_{k_{n}}}{a_{k_{n}+1}-a_{k_{n}}}: a_{k} \in A\right\} .
$$

We have that $h_{n}(\mathbf{Z}(n))=A(n)$ for each $n$. Since $\left(q_{n}-p_{n}\right)$ is a nonzero integer we have that $\mathbf{Z}$ is a subset of $\mathbf{Z}(n)$ for each $n$. Note that from the definition of the set $A(n)$ it follows that the largest negative number in $A(n)$ is

That is if $a<0$ and $a \in A(n)$ we have

$$
\frac{a_{k_{n}-1}-a_{k_{n}}}{a_{k_{n}+1}-a_{k_{n}}}
$$

$$
a \leq \frac{a_{k_{n}-1}-a_{k_{n}}}{a_{k_{n}+1}-a_{k_{n}}} .
$$

On the other hand from (2.9) we conclude

$$
\frac{a_{k_{n}-1}-a_{k_{n}}}{a_{k_{n}+1}-a_{k_{n}}} \rightarrow-\infty, n \rightarrow \infty \text {. }
$$


Therefore we have the following situation. We have the normalized sequence of $K$-quasiconformal maps $h_{n}$ which satisfies that either $0 \leq h_{n}(p)$ or

$$
h_{n}(p) \leq \frac{a_{k_{n}-1}-a_{k_{n}}}{a_{k_{n}+1}-a_{k_{n}}}
$$

for every $p \in \mathbf{Z}$. By passing to a subsequence if necessary we find the $K$ quasiconformal limit map $h: \mathbf{C} \rightarrow \mathbf{C}$. By (2.11) we have that $h$ satisfies that $0 \leq h_{n}(p)$ for every $p \in \mathbf{Z}$. Now we show that the quasiconformal map $h$ with the above properties can not exist. Let

$$
H_{t}(z)=\frac{h(t z)}{h(t)}, z \in \mathbf{C}
$$

where $t>0 . H_{t}$ is a $\mathrm{K}$-quasiconformal map for every $t>0$ and $H_{t}(0)=0$, $H_{t}(1)=1$. Moreover we have that $0 \leq H_{t}(q)$ for every $q=\frac{p}{t}$ where $p$ is an arbitrary integer. Let $t \rightarrow \infty$. Then by passing to a subsequence if necessary, the sequence $H_{t}$ converges to the $K$-quasiconformal map $H$ : $\mathbf{C} \rightarrow \mathbf{C}$ which satisfies the condition $0 \leq H(r)$ for every real number $r$ and $H(0)=0, H(1)=1$. This is clearly impossible since the map $H$ extends to a homeomorphism of the Riemann sphere onto itself.

\section{Proof of Theorem 1.1.}

We split the proof into several lemmas. The notation we introduce remains valid throughout the entire section. As in the previous section $R$ is given by $R=\mathbf{C} \backslash \mathbf{Z}$. Also $\sigma^{2}|d z|^{2}$ denote the density of the hyperbolic metric on $R$. The domain $R$ has an important property that it is invariant under the horizontal translations for integers vectors. That implies that the hyperbolic density $\sigma^{2}|d z|^{2}$ is invariant under those translations. In this section we work exclusively with planar domains and we will drop the notation $d z^{2}$ for denoting quadratic differentials. Define the differential $\varphi_{w}$ by

$$
\varphi_{w}(z)=\frac{1}{(z-w)(z-w-1)(z-w-2)} .
$$

Clearly for each $w \in \mathbf{Z}$ we have $\varphi_{w} \in B Q D(R)$ as a function of $z \in R$. Moreover all $\varphi_{w}$ have the same Bers norm. Let $\left\{p_{k}\right\}, k \geq 1$, be an increasing sequence of nonnegative integers and $C>0$ (we assume that the constant $C$ is an arbitrary positive number, and its exact value is to be chosen later). Set

$$
\psi(z)=C \sum_{k=1}^{\infty} k \varphi_{p_{k}}(z) .
$$


The idea of our proof is to show that there is a constant $C$ and a sequence $\left\{p_{k}\right\}$ with the above properties, such that there exists a surjective harmonic diffeomorphism of $R$ onto some plane domain $S$, and where the Hopf differential of $f$ equals $\psi$, and such that the planar domains $R$ and $S$ are not quasiconformally related.

First we have to properly define the sequence $\left\{p_{k}\right\}$ and the constant $C>0$.

Remark 3.1. One can explicitly determine the sequence $\left\{p_{k}\right\}$ such that all needed conditions are satisfied. But that would involve proving some rather complicated estimates on the solution $\omega$ of the equation (2.3) for the differential $\psi$ from $Q D(R)$. One "advantage" of the inductive approach is that we can see better what lies in the heart of the matter. On the other hand an explicit example would give information of how fast the sequence $\left\{p_{k}\right\}$ grows.

So instead of directly defining the sequence $\left\{p_{k}\right\}$ we will define it inductively. Let $n \in \mathbf{N}$ be a fixed number. Assume that $p_{k}, 1 \leq k \leq n$ is a set of integers which are indexed in the increasing order. For some $C>0$ we define

$$
\psi_{n}(z)=C \sum_{k=1}^{n} k \varphi_{p_{k}}(z)
$$

We have $\psi_{n} \in B Q D(R)$. Let $\omega_{n}$ be the solution of equation (2.3) (from Proposition 2.1) for $\psi_{n} \in B Q D(R)$ and let

$$
\mu_{n}=\frac{\overline{\psi_{n}}}{\omega_{n}^{2}} .
$$

Suppose in addition that the following two conditionds are satisfied

$$
\int_{\{|z|<r\}} \frac{1}{1-\left|\mu_{n}\right|} \leq 10 r^{2}\left(2-\frac{1}{n}\right),
$$

for each $r>0$, and

$$
\int_{\mathbf{C}}\left|\varphi_{p_{k}}\right| \frac{\left|1-\mu_{n} \frac{\varphi_{p_{k}}}{\left|\varphi_{p_{k}}\right|}\right|^{2}}{1-\left|\mu_{n}\right|^{2}} \leq 2\left(2-\frac{1}{n}\right) \int_{\mathbf{C}}\left|\varphi_{p_{k}}\right| \frac{1-\left|\mu_{n}\right|}{1+\left|\mu_{n}\right|},
$$

for each $p_{k}, 1 \leq k \leq n$. The follwoing lemma is the main step toward the construction of the differential $\psi$. 
Lemma 3.1. Suppose that the differential $\psi_{n}$ which is given by (3.1) satisfies (3.3) and (3.4). Then there exists a large enough integer $p_{0}$ which is greater than $p_{k}$ for each $1 \leq k \leq n$, such that for each $p>p_{0}$ the following holds. Set

$$
\psi_{n, p}(z)=\psi_{n}(z)+C(n+1) \varphi_{p}(z)
$$

Denote by $\omega_{n, p}$ the solution of the equation (2.3) for $\psi_{n, p}$ and set

$$
\mu_{n, p}=\frac{\overline{\psi_{n, p}}}{\omega_{n, p}^{2}}
$$

Then $\mu_{n, p}$ satisfies

$$
\int_{\{|z|<r\}} \frac{1}{1-\left|\mu_{n, p}\right|} \leq 10 r^{2}\left(2-\frac{1}{n+1}\right)
$$

for each $r>0$, and

$$
\int_{\mathbf{C}}\left|\varphi_{p_{k}}\right| \frac{\left|1-\mu_{n, p} \frac{\varphi_{p_{k}}}{\left|\varphi_{p_{k}}\right|}\right|^{2}}{1-\left|\mu_{n, p}\right|^{2}} \leq 2\left(2-\frac{1}{n+1}\right) \int_{\mathbf{C}}\left|\varphi_{p_{k}}\right| \frac{1-\left|\mu_{n, p}\right|}{1+\left|\mu_{n, p}\right|}
$$

for each $p_{k}, 1 \leq k \leq n$, and also

$$
\int_{\mathbf{C}}\left|\varphi_{p}\right| \frac{\left|1-\mu_{n, p} \frac{\varphi_{p}}{\left|\varphi_{p}\right|}\right|^{2}}{1-\left|\mu_{n, p}\right|^{2}} \leq 2\left(2-\frac{1}{n+1}\right) \int_{\mathbf{C}}\left|\varphi_{p}\right| \frac{1-\left|\mu_{n, p}\right|}{1+\left|\mu_{n, p}\right|} .
$$

Proof. We first show that there is a large enough integer $p_{0}$ such that for any $p>p_{0}$ the conditions (3.7) and (3.8) are satisfied.

Let $p \rightarrow \infty$. Then we conclude that that $\psi_{n, p} \rightarrow \psi_{n}$ uniformly on compact sets on $R$. By Lemma 2.1 we have that the corresponding solutions $\omega_{n, p}$ of the equeation (2.3) converge to $\omega_{n}$ uniformly on compact set in $R$. From the definition (3.5) we see that the same conclusion holds for $\mu_{n, p}$ and $\mu_{n}$. Now $\varphi_{n}$ and each $\varphi_{n, p}$ are meromorphic function with at most first order poles at real integer points in the complex plane. Consequently it follwos from (3.5) and Proposition 2.1 that $\mu_{n, p}(z)$ and $\mu_{n}(z)$ tend to zero when $z$ tends to the real integer point in the boundary of $R$. Therefore $\mu_{n, p}$ converges to $\mu_{n}$ uniformly on compact sets in C. Since

$$
\left(2-\frac{1}{n}\right)<\left(2-\frac{1}{n+1}\right)
$$


we find that $\mu_{n, p}$ satisfies (3.7) for each $p$ large enough because $\mu_{n}$ satisfies (3.4).

We show that (3.8) holds for a large enough integer $p$. When $p \rightarrow \infty$, $\psi_{n, p}(z) \rightarrow C(n+1) \varphi_{p}(z)$ uniformly on $\mathbf{D}(p, t)$ for fixed $t>0$, where $\mathbf{D}(p, t)$ denotes the disc of radius $t>0$ centered at the point $p$. Define $\nu_{n, p}$ by

$$
\nu_{n, p}=\frac{C(n+1) \overline{\varphi_{p}}}{\gamma_{n, p}^{2}}
$$

where $\gamma_{n, p}$ is the solution to the equation (2.3) for the differential $C(n+1) \varphi_{p}$. Note that

$$
\mu_{n, p}=\left|\mu_{n, p}\right| \frac{\left|\psi_{n, p}\right|}{\psi_{n, p}}, \quad \nu_{n, p}=\left|\nu_{n, p}\right| \frac{\left|\varphi_{n, p}\right|}{\varphi_{n, p}} .
$$

Again by applying Lemma 2.1 we conclude that $\mu_{n, p} \rightarrow \nu_{n, p}$ uniformly on $\mathbf{D}(p, t)$, for fixed $t>0$ and when $p \rightarrow \infty$. This means that for every $\epsilon>0$ there exists $p_{\epsilon}$ such that $\left|\mu_{n, p}(z)-\nu_{n, p}(z)\right|<\epsilon$ for $p>p_{\epsilon}$ and for every $z \in \mathbf{D}(p, t)$.

To prove (3.8) it is enough to prove

$$
\int_{\mathbf{C}}\left|\varphi_{p}\right|\left|\frac{\left|1-\mu_{n, p} \frac{\varphi_{p}}{\left|\varphi_{p}\right|}\right|^{2}}{1-\left|\mu_{n, p}\right|^{2}}-\frac{1-\left|\mu_{n, p}\right|}{1+\left|\mu_{n, p}\right|}\right| \rightarrow 0, \quad p \rightarrow \infty .
$$

because

$$
\int_{\mathbf{C}}\left|\varphi_{p}\right| \frac{1-\left|\mu_{n, p}\right|}{1+\left|\mu_{n, p}\right|} \rightarrow \text { const. }>0, \quad p \rightarrow \infty .
$$

We have that $\mu_{n, p}(z) \rightarrow \nu_{n, p}$ when $p \rightarrow \infty$ uniformly on $\mathbf{D}(p, t)$, for fixed $t$. Then we obtain

$$
\begin{aligned}
& \int_{\mathbf{C}}\left|\varphi_{p}\right|\left|\frac{\left|1-\mu_{n, p} \frac{\varphi_{p}}{\left|\varphi_{p}\right|}\right|^{2}}{1-\left|\mu_{n, p}\right|^{2}}-\frac{1-\left|\mu_{n, p}\right|}{1+\left|\mu_{n, p}\right|}\right| \\
& =\int_{\mathbf{D}(p, t)}\left|\varphi_{p}\right|\left|\frac{\left|1-\mu_{n, p} \frac{\varphi_{p}}{\mid \varphi_{p}}\right|^{2}}{1-\left|\mu_{n, p}\right|^{2}}-\frac{1-\left|\mu_{n, p}\right|}{1+\left|\mu_{n, p}\right|}\right| \\
& +\int_{\mathbf{C} \backslash \mathbf{D}(p, t)}\left|\varphi_{p}\right|\left|\frac{\left|1-\mu_{n, p} \frac{\varphi_{p}}{\left|\varphi_{p}\right|}\right|^{2}}{1-\left|\mu_{n, p}\right|^{2}}-\frac{1-\left|\mu_{n, p}\right|}{1+\left|\mu_{n, p}\right|}\right| .
\end{aligned}
$$


It is clear that the above integral defined over $\mathbf{D}(p, t)$ tends to zero when $p \rightarrow \infty$ for fixed $t$. On the other hand by a change of variables we obtain

$$
\int_{\mathbf{C} \backslash \mathbf{D}(p, t)}\left|\varphi_{p}\right|=\int_{\mathbf{C} \backslash \mathbf{D}(0, t)}\left|\varphi_{0}\right|,
$$

which shows that

$$
\int_{\mathbf{C} \backslash \mathbf{D}(p, t)}\left|\varphi_{p}\right|\left|\frac{\left|1-\mu_{n, p} \frac{\varphi_{p}}{\left|\varphi_{p}\right|}\right|^{2}}{1-\left|\mu_{n, p}\right|^{2}}-\frac{1-\left|\mu_{n, p}\right|}{1+\left|\mu_{n, p}\right|}\right| \rightarrow 0,
$$

uniformly in $p$ when $t \rightarrow \infty$. Therefore we proved that (3.8) holds for a large enough $p$.

The second part of the proof is to show that (3.6) holds for a large enough integer $p$. Suppose that this is not true. Then we can choose an increasing sequence of integers $p_{m}, m \in \mathbf{N}$ and a sequence of positive numbers $r_{m}$ such that

$$
10 r_{m}^{2}\left(2-\frac{1}{n+1}\right)<\int_{\left\{|z|<r_{m}\right\}} \frac{1}{1-\left|\mu_{n, p_{m} \mid}\right|},
$$

for each $r_{m}, m \in \mathbf{N}$. There are two options. First option is that sequence $r_{m}$ stays bounded when $m \rightarrow \infty$, say $r_{m}<r_{0}$. Similarly as we did before, we apply Lemma 2.1 and from the fact that $\psi_{n, p_{m}} \rightarrow \psi_{n}$ when $m \rightarrow \infty$ we see that (3.6) follows from (3.3). This contradicts the assumption (3.9). Second option is that sequence $r_{m}$ is not bounded. By passing to a subsequence if necessary we may assume that $r_{m} \rightarrow \infty$. Let us obtain the contradiction to the assumption (3.9). Rewrite the definition (3.5) as

$$
\mu_{n, p_{m}}=\frac{\sigma^{-2} \overline{\psi_{n, p_{m}}}}{\sigma^{-2} \omega_{n, p_{m}}}
$$

For each $p_{m}$ the differential $\psi_{n, p_{m}}$ is the linear combination of $n+1$ differentials of the type $\varphi_{w}$ which all have the same Bers norm. Also the largest multiplicative coefficient in this linear combination is $C(n+1)$. This yields that the following estiamte of the Bers norm $\psi_{n, p}$. We have

$$
\left\|\psi_{n, p}\right\|_{B} \leq C(n+1)^{2}\left\|\varphi_{1}\right\|_{B},
$$

regardless of what point $p$ we choose. 
Let $\sigma_{1}^{2}|d z|^{2}$ denote the density of the hyperbilic metric on the domain $\mathbf{C} \backslash\{1,2,3\}$. We have

$$
\sigma_{1}^{-2}\left|\varphi_{1}(z)\right| \rightarrow 0, \operatorname{Im}(z) \rightarrow \infty
$$

From the maximum principle for the hyperbolic metric and from the fact that the domain $R$ is contained in the domain $\mathbf{C} \backslash\{1,2,3\}$, we conclude that $\sigma_{1}(z) \leq \sigma(z)$ at every point $z \in R$. We find

$$
\sigma^{-2}\left|\varphi_{1}(z)\right| \rightarrow 0, \operatorname{Im}(z) \rightarrow \infty .
$$

Together the above relations give

$$
\sigma^{-2}\left|\psi_{n, p}(z)\right| \rightarrow 0, \operatorname{Im}(z) \rightarrow \infty
$$

By the definition (3.10) and the fact that

$$
1 \leq \sigma^{-2} \omega_{n, p}^{2},
$$

we conclude that there is a constant $L_{n}>0$ such that

$$
\left|\mu_{n, p_{m}}(z)\right|<\frac{1}{2},
$$

for each $p_{m}$ and for each $z$ which satisfies that $|\mathcal{I} \mathbb{I}(z)|>L$.

As we already showed, the sequence of differentials $\psi_{n, p_{m}}$ has uniformly bounded Bers norm (the bound is independent of $p_{m}$ ). Then it is well known (see [24], [4], [6]) that there is a uniform bound on the modulus of the dilatation $\mu_{n, p_{m}}$. That is,

$$
\left|\mu_{n, p_{m}}\right| \leq q<1
$$

for some constant $0<q<1$. Set

$$
A_{m}=\left\{|z|<r_{m}\right\} \cap\{z \in \mathbf{C}:|\mathcal{I} m z|>L\} .
$$

Using (3.11) and the above uniform estimate $\left|\mu_{n, p}\right| \leq q$, we estimate the integral from (3.6) as

$$
\begin{aligned}
\int_{\left\{|z|<r_{m}\right\}} \frac{1}{1-\left|\mu_{n, p_{m}}\right|} & \leq \int_{\left\{|z|<r_{m}\right\} \backslash A_{m}} \frac{1}{1-\left|\mu_{n, p_{m}}\right|}+\int_{A_{m}} \frac{1}{1-\left|\mu_{n, p_{m}}\right|} \\
& <\frac{1}{1-q} L r_{m}+2 \pi r_{m}^{2}=2 \pi r_{m}^{2}+O\left(r_{m}\right) .
\end{aligned}
$$

Since the assumption was that $r_{m} \rightarrow \infty$, from the above estimate we derive contradiction with (3.9). This proves that (3.6) holds for every large enough integer $p$. The lemma is proved. 
Lemma 3.2. There exists an increasing sequence of positive integers $\left\{p_{k}\right\}$, $k \in \mathbf{N}$ and a positive constant $C$ such that for $\psi \in Q D(R)$ given by

$$
\psi(z)=C \sum_{k=1}^{\infty} k \varphi_{p_{k}}(z), z \in R
$$

the following holds. Let $\omega$ denote the solution of the equation (2.3) for $\psi$ given by (3.12). Set

$$
\mu=\frac{\bar{\psi}}{\omega^{2}}
$$

Then the following conditions are satisfied

$$
\int_{\{|z|<r\}} \frac{1}{1-|\mu|} \leq 20 r^{2}
$$

for each $r>0$,

$$
\int_{\mathbf{C}}\left|\varphi_{p_{k}}\right| \frac{\left|1-\mu \frac{\varphi_{p_{k}}}{\left|\varphi_{p_{k}}\right|}\right|^{2}}{1-|\mu|^{2}} \leq 4 \int_{\mathbf{C}}\left|\varphi_{p_{k}}\right| \frac{1-|\mu|}{1+|\mu|}
$$

for each $p_{k}, k \in \mathbf{N}$, and

$$
\sigma^{-2}(z)\left|\psi(z)-C k \varphi_{p_{k}}(z)\right| \leq \frac{2}{k}
$$

holds for $z \in\left\{\left|z-p_{k}\right|<k\right\}$ and for $k \in \mathbf{N}$, where $\sigma^{2}|d z|^{2}$ is density of the hyperbolic metric on $R$.

Proof. Set $p_{1}=1$. First, it follows form [22] that we can find the positive constant $C$ such that the following holds

$$
\int_{\{|z|<r\}} \frac{1}{1-\left|\mu_{1}\right|} \leq 10 r^{2}(2-1)
$$

for each $r>0$, and

$$
\int_{\mathbf{C}}\left|\varphi_{p_{1}}\right| \frac{\left|1-\mu_{1} \frac{\varphi_{p_{1}}}{\left|\varphi_{p_{1}}\right|}\right|^{2}}{1-\left|\mu_{1}\right|^{2}} \leq 2\left(2-\frac{1}{1}\right) \int_{\mathbf{C}}\left|\varphi_{p_{1}}\right| \frac{1-\left|\mu_{1}\right|}{1+\left|\mu_{1}\right|} .
$$


Now we consecutively apply Lemma 3.1. At the $n-t h$ step, and for each $n \in \mathbf{N}$, we can choose a positive integer $p_{n}$ such that for each $1 \leq k \leq n$ and for $\mu_{n}$ given by (3.2) we have that the following relation hold

$$
\int_{\{|z|<r\}} \frac{1}{1-\left|\mu_{n}\right|} \leq 10 r^{2}\left(2-\frac{1}{n}\right)
$$

for each $r>0$,

$$
\int_{\mathbf{C}}\left|\varphi_{p_{k}}\right| \frac{\left|1-\mu_{n} \frac{\varphi_{p_{k}}}{\left|\varphi_{p_{k}}\right|}\right|^{2}}{1-\left|\mu_{n}\right|^{2}} \leq 2\left(2-\frac{1}{n}\right) \int_{\mathbf{C}}\left|\varphi_{p_{k}}\right| \frac{1-\left|\mu_{n}\right|}{1+\left|\mu_{n}\right|},
$$

for each $p_{k}, 1 \leq k \leq n$. If we return to the statement and the proof of Lemma 3.1 , we see that we have to choose $p_{n}$ to be large enough, but on the other hand there is no bound on how large $p_{n}$ has to be. Because of this, at the each step of the way we can take $p_{n}$ large enough such that the inequality

$$
\sigma^{-2}(z)\left|\psi_{n}(z)-C k \varphi_{p_{k}}(z)\right| \leq\left(2-\frac{1}{k}\right) \frac{1}{k}
$$

holds for $z \in\left\{\left|z-p_{k}\right|<k\right\}$ and each $1 \leq k \leq n$. With this choice we have arranged two things. First, we have that the sequence of differentials $\psi_{n}$ given by (3.1) converges uniformly to $\psi$ on compact sets in $R$ and in particular $\psi$ is well defined. Second we have that

$$
\sigma^{-2}(z)\left|\psi(z)-C k \varphi_{p_{k}}(z)\right| \leq \frac{2}{k}
$$

holds for $z \in\left\{\left|z-p_{k}\right|<k\right\}$ and for each $k \in \mathbf{N}$.

We now show that the sequence $\left\{p_{k}\right\}$ determined by (3.15) and (3.16) satisfies the rest of the assumption of this lemma. By applying Lemma 2.1 once again we see that $\mu_{n} \rightarrow \mu$ uniformly on compact sets in $R$ (and consequently on compact sets in C). Now (3.13) and (3.14) follow from (3.15) and (3.16) respectively, once we let $n \rightarrow \infty$.

Lemma 3.3. Assume that $\psi \in Q D(R)$ and $\mu$ are given by the previous lemma. Then there is a planar domain $S$ and a surjective harmonic diffeomorphism $f: R \rightarrow S$ such that $\operatorname{Hop} f(f)=\psi$ and Belt $(f)=\mu$. Moreover the diffeomorphism $f$ preserves the real line $\mathbf{R}$. 
Proof. Let $S_{n}$ be the planar domain for which $f_{n}$ is the harmonic diffeomorphism $f_{n}: R \rightarrow S_{n}$ given by Proposition 2.2 where

$$
\operatorname{Hopf}\left(f_{n}\right)=\psi_{n} .
$$

The differential $\psi_{n}$ has the form given by (3.1) where $C>0$ and $\left\{p_{k}\right\}$, $1 \leq k \leq n$ are determined by Lemma 3.1 and Lemma 3.2. Also we may assume that $f_{n}(0)=0$ and $f_{n}(1)=1$. For a moment we consider $f_{n}$ just as a quasiconformal diffeomorphism and we can forget that $f_{n}$ is a harmonic map. Note that the map $f_{n}$ extends to a quasiconformal diffeomorphism of the complex plane onto itself. We have that $\mu_{n}$ is a Beltrami dilatation of $f_{n}$. The Hopf differential $\psi_{n}$ takes the real values on the real line (except possibly at points from $\mathbf{Z}$ where it has at most first order poles). Therefore the Beltrami dilatation $\mu_{n}$ satisfies the symmetry condition and $f_{n}$ preserves the real line (moreover $f_{n}$ maps a symmetric points onto the symmetric points with respect to $\mathbf{R}$ ). From the proof of Lemma 3.2 we know that $\mu_{n}$ converges to $\mu$ uniformly on compact sets in $\mathbf{C}$. Therefore, by passing onto a subsequence if necessary, we find that on each compact set in $\mathbf{C}$ the sequence $f_{n}$ converges to a quasiconformal diffeomorphism $f$ whose Beltrami dilatation is $\mu$. In general $f$ is a diffeomorphism of the complex plane into itself because its Beltrami dilatation is a continuous function $\mu$ (see [1], [22]). But from (3.16) we see that $\mu$ satisfies the assumptions of Corollary 2.2 and by existence and uniqueness part of this corollary we conclude that the diffeomorphism $f$ is surjective map of the complex plane onto itself. Also we see that $f$ preserves the real line (it is also symmetric with respect to the real line same as each $f_{n}$ ).

It remains to find the domain $S$ and to show that $f$ is a surjective harmonic diffeomorphism of $R$ onto $S$. Simply take $S=f(R)$. It is clear that the sequence of the domains $S_{n}$ converges to the domain $S$ in the Hausdorff metric. This implies that $\rho_{n}^{2}|d z|^{2} \rightarrow \rho^{2}|d z|^{2}$ on compact sets in upper and lower half plane (this convergence holds as well on the compact sets of the real line which do not contain boundary points of $S$ and $S_{n}$ when $n$ is large). Here $\rho_{n}^{2}|d z|^{2}$ denotes the density of the hyperbolic metric on $S_{n}$. Since $f_{n}$ and $f$ are diffeomorphisms, and locally uniformly quasiconformal maps, and since $\mu_{n} \rightarrow \mu$ locally uniformlly on $R$, it is known (see [1], [13]) that the sequence of gradients of the maps $f_{n}$ converges locally uniformly to the gradient of $f$ on compact subsets in $R$. From all this we see that the Hopf differential of $f$ is equal $\psi$, that is

$$
H o p f(f)=\left(\rho_{n}^{2} \circ f\right) f_{z} \overline{\left(f_{\bar{z}}\right)}=\psi .
$$


On the other hand it is well known (see [21]) that a diffeomorphism defined on a Riemann surfaces is harmonic if and only if its Hopf differential is a holomorphic quadratic differential. This shows that $f$ is harmonic and the proof of the lemma is complete.

Lemma 3.4. The domains $R$ and $S$ defined above are not quasiconformally related.

Proof. We apply Lemma 2.1. First we have to prove that in this case the assumptions of Lemma 2.1 are satisfied.

Take a point $p_{n}$ from the sequence $\left\{p_{k}\right\}, k \in \mathbf{N}$. Set

$$
a_{n}=f\left(p_{n}\right), b_{n}=f\left(p_{n}+1\right), c_{n}=f\left(p_{n}+2\right) .
$$

Note that $a_{n}<b_{n}<c_{n}$. Define $K_{0}(n)$ as

$$
K_{0}(n)=\inf _{g} K(g)
$$

where $\mathrm{K}(\mathrm{g})$ is maximal dilatation of the quasiconformal map $g$ and the infimum is taken with respect to all quasiconformal maps $g$ which satisfies the boundary condition $a_{n}=g\left(p_{n}\right), b_{n}=g\left(p_{n}+1\right), c_{n}=g\left(p_{n}+2\right)$. First we show that $K_{0}(n) \rightarrow \infty$ when $p_{n} \rightarrow \infty$.

From Lemma 3.2 we conclude that

$$
\sigma^{-2}(z)\left|\psi-C n \varphi_{p_{n}}(z)\right|(z) \rightarrow 0, p_{n} \rightarrow \infty
$$

uniformly for $z \in \mathbf{D}\left(p_{n}, t\right) \cap R$. Here $t>0$ is a fixed number and $\mathbf{D}\left(p_{n}, t\right)$ denotes the disc of radius $t>0$ centered at the point $p_{n}$ (note that the first order poles of $\psi$ do not interfere with this conclusion). In particular we have $\sigma^{2}|\psi|(z) \rightarrow \infty, p_{n} \rightarrow \infty$ uniformly for $z \in \mathbf{D}\left(p_{n}, t\right) \cap R$. Let us show that $|\mu(z)| \rightarrow 1$ when $p_{n} \rightarrow \infty$, uniformly on compact sets in $\mathbf{D}\left(p_{n}, t\right) \cap R$. This conclusion is essentially the consequence of the maximum principle introduced by Wolf and Minsky in [25],[18] (see also [24],[4]). In particular we use techniques from [4] but details may vary.

Fix $z_{n} \in \mathbf{D}\left(p_{n}, t\right) \cap R$ such that for two different points $p_{n}$ and $p_{m}$ we have that $z_{n}-z_{m}$ is a real number such that is $z_{m}$ arises from $z_{n}$ by translating $z_{n}$ for the vector $p_{m}-p_{n}$. Define

$$
P_{n}(z)=\int_{z_{n}}^{z} \psi^{\frac{1}{2}}
$$


where $z \in \mathbf{D}\left(p_{n}, t\right) \cap R$. From (3.17) it follows that $\psi(z)$ does not have zeroes in any compact subset of $\mathbf{D}\left(p_{n}, t\right)$ for $p_{n}$ large enough (because $\varphi_{p_{n}}(z)$ does not have any zeroes in $\mathbf{C}$ ). This implies that $P_{n}$ is well defined on the disc $\mathbf{D}\left(z_{n}, d\right)$, which is contained in $\mathbf{D}\left(p_{n}, t\right)$, for $p_{n}$ large enough. Here we define $d>0$ to be the minimum of the distance from $z_{n}$ to the set of punctures of the domain $R$ and the distance from $z_{n}$ to the boundary of $\mathbf{D}\left(z_{n}, d\right)$. Since $z_{n}-z_{m}=p_{n}-p_{m}$ we see that the distance $d$ is the same for every $z_{n}$. Function $P_{n}$ is known as the natural parameter for the differentials $\psi$. By applying the Köebe and the Bloch theorems (in the same way it has been done in [4]) we easily find an open set $U_{n}$ contained in $\mathbf{D}\left(z_{n}, d\right)$ such that $P_{n}$ is univalent on $U_{n}, P_{n}\left(z_{n}\right)=0$ and $P_{n}\left(U_{n}\right)=\mathbf{D}\left(0, r_{n}\right) . \mathbf{D}\left(0, r_{n}\right)$ is the disc centered at 0 of radius $r_{n}$. In particular since

$$
\left|P_{n}^{\prime}\left(z_{n}\right)\right|=\left|\psi\left(z_{n}\right)\right|^{2} \rightarrow \infty, p_{n} \rightarrow \infty
$$

from the Köebe theorem and the fact that the Euclidean radius of $U_{n}$ is uniformly bounded by $t$, we conclude that $r_{n} \rightarrow \infty$ when $p_{n} \rightarrow \infty$. Next define $\nu_{n}(w), w \in \mathbf{D}\left(0, r_{n}\right)$ such that

$$
\nu_{n}\left(P_{n}(z)\right) \frac{\overline{P_{n}^{\prime}}}{P_{n}^{\prime}}=\mu(z) .
$$

We show that $\left|\nu_{n}\right|(0) \rightarrow 1, p_{n} \rightarrow \infty$. Note that $\nu_{n}$ satisfies the equation

$$
\Delta \log \frac{1}{\left|\nu_{n}\right|}=\frac{1}{\left|\nu_{n}\right|^{2}}-\left|\nu_{n}\right|^{2}
$$

on $\mathbf{D}\left(0, r_{n}\right)$. By Proposition 2.1 and Proposition 2.2 we know that there exists function $\eta_{n}$ defined on $\mathbf{D}\left(0, r_{n}\right)$ which satisfies

$$
\Delta \log \frac{1}{\left|\eta_{n}\right|}=\frac{1}{\left|\eta_{n}\right|^{2}}-\left|\eta_{n}\right|^{2}
$$

and such that the metric space $\left(\mathbf{D}\left(0, r_{n}\right), \frac{1}{\left|\eta_{n}\right|}|d w|^{2}\right)$ is a complete Riemannian surface. From the maximum principle proved in [24] (see also [22], [10]) it follows that $\left|\eta_{n}\right| \leq\left|\nu_{n}\right|$ at every point of $\mathbf{D}\left(0, r_{n}\right)$. On the other hand since $r_{n} \rightarrow \infty$ when $p_{n} \rightarrow \infty$ we know (see [21],[4], [23]) that $\left|\eta_{n}(0)\right| \rightarrow 1$. Therefore we proved that for every point $z_{n} \in \mathbf{D}\left(p_{n}, t\right)$ we have $\left|\mu\left(z_{n}\right)\right| \rightarrow 1$ when $p_{n} \rightarrow \infty$.

Note that the diffeomorphism $f$ and the quasiconformal maps $g$ defined above take the same values at the points $p_{n}, p_{n}+1, p_{n}+2$. Therefore we 
may apply the fundamental form of the Reich-Strebel inequality (also called the Main inequality, see [19], [9]) to the maps $f$ and $g$ and to the quadratic differential $\varphi_{p_{n}}$. The differential $\varphi_{p_{n}}$ has only first order poles at the points $p_{n}, p_{n}+1, p_{n}+2, \infty$, as the differential on the Riemann sphere. In this case the Fundamental inequality has the form

$$
\frac{1}{K_{0}(n)} \int_{\mathbf{C}}\left|\varphi_{p_{n}}\right| \leq \int_{\mathbf{C}}\left|\varphi_{p_{n}}\right| \frac{\left|1-\mu \frac{\varphi_{p_{n}}}{\mid \varphi_{p_{n}}}\right|^{2}}{1-|\mu|^{2}} .
$$

Remark 3.2. Note that the while $g$ is a K-quasiconformal map, the diffeomorphism $f$ is not a quasiconformal map. Therefore in order to apply the Main inequality in this setting we use the results (see [17],[15]) where the Main inequality was proved in this general setting without the restriction that the mappings we apply the inequality to, have to be necessarily quasiconformal(they still have to be univalent and quasiconformal on every compact subset of the underlying domain).

Note that

$$
0<\int_{\mathbf{C}}\left|\varphi_{p_{n}}\right|=\text { const. }<\infty
$$

for each $p_{n}$. Also we have

$$
0<\int_{\mathbf{C} \backslash \mathbf{D}\left(p_{n}, t\right)}\left|\varphi_{p_{n}}\right| \rightarrow 0, t \rightarrow \infty
$$

uniformly for every point $p_{n}$. Combining this with (3.16) and the fact that $|\mu| \rightarrow 1$ uniformlly in compact sets in $\mathbf{D}\left(p_{n}, t\right)$, we conclude that $K_{0}(n) \rightarrow$ $\infty$.

In order to apply Lemma 2.1 to the harmonic diffeomorphism $f: R \rightarrow S$ we need to show that the supremum $\mathbf{s}$ defined by the formula (2.8) equals $\infty$. That directly follows from the fact that $K_{0}(n) \rightarrow \infty$. Namely if $\mathbf{s}$ is a real number (and not equal $\infty$ ) we would have that the sequence

$$
\left|\frac{a_{n}-b_{n}}{b_{n}-c_{n}}\right|+\left|\frac{b_{n}-c_{n}}{a_{n}-b_{n}}\right|
$$

remains bounded when $n \rightarrow \infty$. In this case it is elementary to directly construct the sequence of $K_{1}$-quasiconformal maps $\left\{g_{n}\right\}$ which satisfy

$$
a_{n}=g_{n}\left(p_{n}\right), b_{n}=g_{n}\left(p_{n}+1\right), c_{n}=g_{n}\left(p_{n}+2\right),
$$


and where $K_{1}$ is a constant which depends only upon the upper bound of the above expression . But this would imply $K_{0}(n) \leq K_{1}<\infty$ which is a contradiction.

Theorem 1.1 is proved.

\section{Harmonic diffeomorphisms between surfaces of finite topological type.}

In this section we prove Theorem 1.2. The notation we introduce below remains valid throughout the entire section. We already mentioned that every Riemann surface $R$ of finite topological type is conformally equivalent to the Riemann surface obtained from some closed surface $M$ of the finite genus $\mathrm{g}$ with $n$ discs $\mathbf{D}_{i}, 1 \leq i \leq n$ and $m$ punctures $p_{j}, 1 \leq j \leq m$ removed. That is

$$
R=M \backslash\left(\cup_{i=1}^{n} \mathbf{D}_{i}\right) \backslash\left(\cup_{j=1}^{m} p_{j}\right) .
$$

Suppose that $f: R \rightarrow S$ is a surjective harmonic diffepmorphism and assume that $\left(R,|\partial f|^{2}|d z|^{2}\right)$ is a complete Riemannian surface. Set $H o p f(f)=\psi d z^{2}$. We note that $|\partial f|$ is the solution of the equation (2.3) for the differential $\psi d z^{2}$. The Riemann surface $S$ is of finite topological type as well. Moreover $S$ is represented as

$$
S=N \backslash\left(\cup_{i=1}^{n_{1}} \mathbf{D}^{\mathbf{1}}{ }_{i}\right) \backslash\left(\cup_{j=1}^{m_{1}} q_{j}\right),
$$

where $N$ is some closed Riemann surface of the same genus $\mathbf{g}$ and $\mathbf{D}^{\mathbf{1}}{ }_{i}$, $1 \leq i \leq n_{1}, q_{j}, 1 \leq j \leq m_{1}$ are respectively the discs and punctured removed form $N$. Since $f$ is a homeomorphism we have that $n+m=n_{1}+m_{1}$. The necessary and sufficient condition for surfaces $R$ and $S$ given by (4.1) and (4.2) to be quasiconformally related (see [1], [13]) is that $n=n_{1}$ and $m=m_{1}$, and that the surfaces $M$ and $N$ share the same genus $\mathrm{g}$. So, in order to prove Theorem 1.2 we need to show that under the given assumptions we have that $n=n_{1}$ and $m=m_{1}$. To do so we need to investigate the behavior of the map $f$ near removed discs $\mathbf{D}_{i}$ and punctures $p_{j}$.

Let $U_{1}$ be the subset of $R$ such that $U_{1}$ is conformally equivalent to the annulus $P(r)=\{r<|z|<1\}$ in the complex plane for some $0<r<1$ and such that the set $U_{1} \cup \mathrm{D}_{1}$ is a simply connected domain in the closed surface $M$. Let $V_{1}=f\left(U_{1}\right)$. Since $f$ is a homeomorphism we can find either disc $\mathbf{D}_{1}^{1}$ or a puncture $q_{1}$ from the representation (4.2) such that union of $V_{1}$ with 
one of these two is a simply connected domain. This simply means that $f$ maps the neighborhood of $\mathbf{D}_{1}$ either onto a neighborhood of some disc or a neighborhood of some puncture from the representation (4.2). In the first case $V_{1}$ is conformally equivalent to an annulus and in the second case $V_{1}$ is conformally equivalent to the punctured unit disc $\mathbf{D}^{*}=\{0<|z|<1\}$. Since $f$ is a harmonic map it turns out that precisely one of these two cases can occur.

Lemma 4.1. With the notation and assumptions stated above we have that the image $V_{1}$ of the domain $U_{1}$ is also conformally equivalent to an annulus.

Proof. Once again we denote by $\sigma^{2}|d z|^{2}$ the density of the hyperbolic metric on $R$ where $z$ is the local parameter. Note that in this case $R$ is a hyperbolic Riemann surface since we removed at least one disc from the closed surface $M$ to obtain the Riemann surface $R$. Denote by $\rho^{2}|d w|^{2}$ the hyperbolic metric on $S$ (of course $S$ is a hyperbolic Riemann surface and the map $f$ is harmonic with respect to the metric $\rho$ ).

Suppose that $V_{1}$ is conformally equivalent to the punctured unit disc $\mathbf{D}^{*}$ and let $q_{1}$ be the puncture such that $V_{1} \cup q_{1}$ is a simply connected domain in $N$. Then we have that the hyperbolic area of $V_{1}$ is finite. Recall that $|\partial f|$ satisfies the Bochner identity (2.1). An equivalent form of (2.1) is the following formula

$$
\Delta \log (|\partial f|)=|\partial f|^{2}-|\bar{\partial} f|^{2}
$$

The right side of the above formula is exactly the Jacobian of $f$ taken with respect to the hyperbolic metric on $S$. We have

$$
\int_{U_{1}}|\partial f|^{2}-|\bar{\partial} f|^{2}=\int_{U_{1}} \Delta \log (|\partial f|)<\infty .
$$

Let $\alpha: U_{1} \rightarrow P(t)$ be the conformal map such that $|\alpha(p)| \rightarrow 1$ when $p$ approaches the boundary of $R$. Denote by $\gamma_{t}$ the pull back of the circle $|z|=t$ for every $r<t<1$. Set

$$
I(t)=\frac{1}{2 t \pi} \int_{\gamma_{t}} \log |\partial f|
$$

For $t_{0}<t$, the Green's formula yields

$$
t I^{\prime}(t)-t_{0} I^{\prime}\left(t_{0}\right)=\frac{1}{2 \pi} \int_{G(t)} \Delta \log (|\partial f|),
$$


where $G(t)$ is the region bounded by $\gamma_{t_{0}}$ and $\gamma_{t}$ and $I^{\prime}(t)$ is the derivative of $I(t)$ taken with respect to the real variable $t$. From (4.3) and the above relation it follows that $I^{\prime}(t)$ remains bounded when $t \rightarrow 1$. But this implies that $I(t)$ remains bounded when $t \rightarrow 1$. That is impossible because the inequality $1<\sigma^{-2}|\partial f|$ directly implies $I(t) \rightarrow \infty, t \rightarrow 1$. This proves the contradiction and we conclude that $V_{1}$ has to be conformally equivalent to an annulus.

Next we consider the case when $U_{1}$ is the subdomain of $R$ conformally equivalent to the punctured unit disc $\mathbf{D}^{*}$. Also there is a point $p_{1}$ from the representation (4.1) and the set $U_{1} \cup \mathbf{D}_{1}$ is a simply connected domain in the closed surface $M$. Let $V_{1}=f\left(U_{1}\right)$. Again we have find that since $f$ is a homeomorphism we can find either a disc $\mathbf{D}_{1}^{1}$ or a puncture $q_{1}$ from the representation (4.2) such that union of $V_{1}$ with one of these two is the simply connected domain in $N$. This simply means that $f$ maps the neighborhood of $p_{1}$ either onto a neighborhood of the disc or a neighborhood of a puncture from the representation (4.2). In the first case $V_{1}$ is conformally equivalent to an annulus and in the second case $V_{1}$ is conformally equivalent to the punctured unit disc $\mathbf{D}^{*}=\{0<|z|<1\}$. In this type of the situation we know that $V_{1}$ can be conformally equivalent to the punctured unit disc but unlike the case we had in Lemma 4.1, without further assumptions we can not exclude the possibility that $V_{1}$ is conformally equivalent to an annulus. We have

Lemma 4.2. With the notation stated above and under the assumptions of Theorem 1.2 we have that $V_{1}$ is conformally equivalent to the punctured disc.

Proof. By $\rho_{1}|d z|^{2}$ we denote the hyperbolic metric on the domain $U_{1}$. Let $g: U_{1} \rightarrow A$ be the harmonic diffeomorphism from Corollary 2.1 such that $H o p f(g)=\psi d z^{2}$. Set $\nu \frac{d \bar{z}}{d z}=\operatorname{Belt}(g)$. By the same corollary we know that $A$ is the Riemann surface conformally equivalent either to an annulus or to the punctured unit disc. Also, both $|\partial g|$ and $|\partial f|$ satisfy the equation (2.3) for the same differential $\psi d z^{2}$. Since $\left(U_{1},|\partial g|^{2}|d z|^{2}\right)$ is a complete Riemannian surface we obtain that the inequalities

$$
|\partial f| \leq|\partial g|,|\bar{\partial} g| \leq|\bar{\partial} f|,
$$

hold at every point of $U_{1}$. Set $\mu \frac{d \bar{z}}{d z}=\operatorname{Belt}(f)$. Form (4.4.) we obtain that

$$
|\nu| \leq|\mu|
$$


hold on $U_{1}$ as well.

Now we prove that the map $g$ is surjective. Let $\gamma$ be an arbitrary smooth curve in $U_{1}$ such that $\gamma$ does not remain in any compact subset of $U_{1}$, that is $\gamma$ has density points on the boundary of $U_{1}$. To prove that $g$ is a surjective map it is enough to show that the hyperbolic length $l_{A}(g(\gamma))$ in $A$ is infinite. The domain $U_{1}$ has the two boundary components, one of them is the point $p_{1}$ and the second one is some simple closed curve $u_{1}$ compactly contained in $R$. The first case we have to consider is when $\gamma$ has density points on $u_{1}$. As a harmonic diffeomorphism the map $f$ is the smooth and quasiconformal in some neighborhood $\tilde{U}_{1}$ of $u_{1}$ which is compactly contained in $R$. Then there is a constant $q<1$ such that $|\nu \leq| \mu \mid \leq q$ holds on $\tilde{U}_{1}$. We have

$$
\begin{aligned}
l_{A}(g(\gamma))=\int_{\gamma}|\partial g d z+\bar{\partial} g d \bar{z}| & =\int_{\gamma}|\partial g|\left|1+\nu \frac{d \bar{z}}{d z}\right||d z| \\
& \geq \int_{\gamma}|\partial g|(1-q)|d z|=\infty .
\end{aligned}
$$

The value of the last integral above is equal $\infty$ because the metric whose density is given by $|\partial g|^{2}|d z|^{2}$ is complete on $U_{1}$.

The second case we have to consider is when the point $p_{1}$ is the density point of the curve $\gamma$. This is the place where we essentially use the assumption that the harmonic diffeomorphism $f: R \rightarrow S$ is surjective. Namely in this case we have that the hyperbolic length $l_{S}(f(\gamma))$ of the curve $f(\gamma)$ in $S$ is infinite. We have

$$
l_{S}(g(\gamma))=\int_{\gamma}|\partial f d z+\bar{\partial} f d \bar{z}|=\infty .
$$

Now we prove the inequality

$$
|\partial f d z+\bar{\partial} f d \bar{z}| \leq|\partial g d z+\bar{\partial} g d \bar{z}|
$$

which immediately yields the conclusion $l_{A}(g(\gamma)) \geq l_{S}(f(\gamma))=\infty$. Combining the fact that

$$
\frac{\bar{\partial} f}{\bar{\partial} g}=\frac{\partial g}{\partial f}
$$

with (4.4), we conclude that

$$
|\partial f|^{2}+|\bar{\partial} f|^{2} \leq|\partial g|^{2}+|\bar{\partial} g|^{2}
$$


holds everywhere in $U_{1}$. After the squaring, the inequality (4.5) becomes the same as the above inequality and therefore (4.5) is proved. The fact that the map $g: U_{1} \rightarrow A$ is surjective is now proved.

By the assumption of Theorem 1.2 (and this lemma) we conclude that the image surface $A$ is conformally equivalent to the punctured unit disc. Let us show that $V_{1}$ has the same property. From (4.4) we derive $J_{f} \leq J_{g}$ where the Jacobians $J_{f}$ and $J_{g}$ are taken with the respect to the hyperbolic metric on $S$ and $A$ respectively. Let $\overline{U_{1}}$ be the neighborhood of the point $p_{1}$ defined by $\overline{U_{1}}=U_{1} \backslash \tilde{U}_{1} . A$ is conformally equivalent to the punctured unit disc and we conclude

$$
\int_{\overline{U_{1}}} J_{g} \leq \infty .
$$

But then we have that the same holds for the map $f$

$$
\frac{\int}{U_{1}} J_{f} \leq \infty .
$$

Since $f$ is surjective, an immediate corollary of the above inequality is that $V_{1}$ is conformally equivalent to the punctured unit disc and we the lemma is proved.

Proof of Theorem 1.2. From Lemma 4.1 we see that the map $f$ maps the neighborhood of the removed disc from (4.1) onto the neighborhood of the removed disc from (4.2). From Lemma 4.2 we have that the same conclusion holds for removed punctures. Therefore we derive that $n=n_{1}$ and $m=m_{1}$ and we conclude that $R$ and $S$ are quasiconformally related since we already know that $M$ and $N$ have the same genus $\mathrm{g}$.

\section{References.}

[1] L. Ahlfors, Lectures on Quasiconformal Mappings, Van Nostrand, Princeton, New Jersey, 1966.

[2] K. Akutagawa and S. Nishikawa, The Gauss map and spacelike surfaces with prescribed mean curvature in Minkowski 3-space, Tohoku Math. J., 42(1) (1990), 67-82. 
[3] K. Akutagawa and A. Tachikawa, Nonexistence results for harmonic maps between noncompact complete Riemannian manifolds, Tokyo J. Math., 16 (1993), 131-145.

[4] I. Anić, V. Marković, and M.Mateljević, Uniformly bounded maximal $\Phi$-disks, Bers spaces and harmonic maps, Proc. Amer. Math. Soc., 10 (2000).

[5] K. Au and T. Wan, Parabolic constant mean curvature spacelike surfaces, Proc. Amer. Math. Soc., 2 (1994), 559-564.

[6] K. Au, L. Tam, and T. Wan, Hopf differentials and the images of harmonic maps, Preprint, 2000.

[7] M. Brakalova and J. Jenkins, On solutions of the Beltrami equation, J. Anal. Math., 76 (1998), 67-92.

[8] H. Choi and A. Treibergs, Gauss maps of spacelike constant mean curvature hypersurfaces of Minkowski space, J. Diff. Geom., 32 (1990), 775-818.

[9] F.P. Gardiner, Teichmüller Theory and Quadratic Differentials, John Wiley and Sons, New York, 1987.

[10] Z-C. Han, Remarks on the geometric behaviour of harmonic maps between surfaces, Proceedings of a Workshop, Minneapolis, May 23-24, 1994.

[11] Z.C. Han, L. Tam, A. Treibergs, and T. Wan, Harmonic maps from the complex plane into surfaces with nonpositive curvature, Commun. Anal. Geom., 3 (1995), 85-114.

[12] J. Jost, Two dimensional geometric variational poblems. John Wiley \& Sons, 1991.

[13] O. Lehto, Univalent functions and Teichmüller spaces, Graduate Texts in Mathematics, 109, Springer, Berlin, 1987.

[14] O. Lehto, and K.I. Virtanen, Quasiconformal mappings in the plane, Springer-Verlag, Berlin-Heidelberg-New York, 1973.

[15] V. Marković, Uniquely extremal quasiconformal mappings and stationary points of the energy integral, Ph.D. Thesis, University of Belgrade, 1998. 
[16] V. Markovic, Harmonic diffeomorphisms between noncompact surfaces and Teichmüller spaces, J. London Math. Soc. (2), 65 (2002) 103-114.

[17] V. Marković and M. Mateljević, New version of the main inequality and uniqueness of harmonic maps, Journal d'Analyse, 1999.

[18] Y. Minsky, Harmonic maps, length and energy in Teichmüller spaces, J. Diff. Geom., 35 (1992), 151-217.

[19] E. Reich and K. Strebel, On quasiconformal mappings which keep the boundary points fixed, Trans. Amer. Math. Soc., 138 (1969).

[20] $\mathrm{R}$. Schoen, The role of harmonic mappings in rigidity and deformation problems.

[21] R. Schoen and S.T. Yau, Lectures on harmonic maps, Conf. Proc. and Lect. Not. in Geometry and Topology, II (1997).

[22] L.F. Tam and T. Wan, Quasiconformal harmonic diffeomorphism and the universal Teichmüller space, J.Differential Geometry, 42 (1995).

[23] L.F. Tam and T. Wan, Harmonic diffeomorphisms into CartanHadamar surfaces with prescribed Hopf differentials, Commun. Anal. Geom, 4 (1994), 593-625.

[24] T. Wan, Constant mean curvature surface, harmonic maps and universal Teichmüller space, J. Diff. Geom., 35 (1992), 643-657.

[25] M. Wolf, The Teichmüller theory of harmonic maps, J. Diff. Geom., 29 (1989), 449-479.

[26] M. Wolf, Infinite energy harmonic maps and degeneration of hyperbolic surfaces in moduli space, J. Diff. Geom., 35 (1991), 487-539.

UNIVERSITY OF WARWICK

INSTITUTE OF MATHEMATICS

CV4 7AL Coventry, UK

E-mail address: markovic@maths.warwick.ac.uk

RECeived November 15, 2000. 